\title{
Recurrent Early Filter Clotting during Continuous Veno-Venous Hemodialysis with Regional Citrate Anticoagulation is Linked to Systemic Thrombin Generation and Heparin Induced Thrombocytopenia Type II: A Retrospective Analysis
}

\begin{abstract}
Khadzhynov $\mathbf{D}^{1 *}$, Slowinski $\mathbf{T}^{1}$, Schreiber $\mathbf{A}^{1}$, Lieker I' ${ }^{1}$ Halleck F', Lehner LJ ${ }^{1}$, Kindgen-Milles $D^{2}$, Eckardt K-U' ${ }^{1}$, Budde $K^{1}$ and Staeck $\mathbf{O}^{1}$

${ }^{1}$ Department of Nephrology and Medical Intensive Care, Charité-Universitätsmedizin Berlin, Berlin, Germany ${ }^{2}$ Department of Anesthesiology/ University Hospital Duesseldorf/Heinrich-Heine-University, Duesseldorf, Germany

*Corresponding author: Dmytro Khadzhynov, Department of Nephrology and Medical Intensive Care, Charité Universitätsmedizin Berlin, Charitéplatz 1, D-10117 Berlin, Germany
\end{abstract}

Received: September 01, 2021; Accepted: September 23, 2021; Published: September 30, 2021

\begin{abstract}
Objective: Regional Citrate Anticoagulation (RCA) for Continuous Renal Replacement Therapy (CRRT) is widely used and leads to an excellent clottingfree filter survival. Despite strict adherence to protocols, in some cases recurrent early filter-clotting occurs. The aim of this observational study was to evaluate the underlying causes and the efficacy of interventions in patients with early recurrent filter-clotting during RCA.
\end{abstract}

Methods: In a retrospective analysis of a cohort of 1183 patients treated with RCA-CRRT we detected 12 patients with early filter-clotting unrelated to protocol violation or any obvious technical or medical reason.

Results: All patients were systemically anticoagulated with low molecular weight or unfractionated heparin for at least $24 \mathrm{~h}$ before initiation of Continuous Veno-Venous Hemodialysis with RCA (RCA-CVVHD). During RCA, all postfilter ionized calcium concentrations were in the target range (mean $0.33 \pm 0.05$ $\mathrm{mmol} / \mathrm{L}$ ). At the time of the first clotting event, thrombocyte counts were $168 \pm 66 /$ $\mathrm{nL}$. After the clotting events, the systemic anticoagulation was switched to argatroban in all patients. With systemic anticoagulation using argatroban filter lifetime of RCA-CVVHD increased significantly $(p<0.001)$ and clotting-events decreased from 0.61 to 0.10 per $24 \mathrm{~h}$. All patients were tested for HIT and $5 / 12$ $(42 \%)$ had a positive test for hep-PF4-antibodies. Application of argatroban significantly reduced early filter-clotting both in HIT-positive patients as well as in HIT-negative patients. At the time of the first clotting event, no patient had clinical signs of thrombosis or thromboembolism. However, during follow up a thromboembolic event occurred in three patients.

Conclusion: In patients with recurrent early filter-clotting despite strict adherence to the citrate protocol undetected HIT or other causes of thrombin activation may be present. Therefore, patients with recurrent early filter clotting in RCA-CVVHD should be screened for HIT or other conditions that may activate thrombin. A significant improvement of filter run-time can be achieved by systemic administration of a thrombin inhibitor both in patients with and without HIT.

Keywords: Continuous veno-venous hemodialysis; Regional citrate anticoagulation; Filter-clotting; Heparin induced thrombocytopenia type 2; Argatroban; Thrombin

\section{Abbreviations}

AKI: Acute Kidney Injury; RCA: Regional Citrate Anticoagulation; CRRT: Continuous Renal Replacement Therapy; CVVHD: Continuous Veno-Venous Hemodialysis; GLA-Domain: Gamma-Carboxyglutamic Domain; HIPA: Heparin-Induced Platelet Aggregation; HIT: Heparin Induced Thrombocytopenia; ICU: Intensive Care Unit; SD: Standard Deviation; IQR: Interquartile Range

\section{Introduction}

Regional Citrate Anticoagulation (RCA) has become a widely used technique in CRRT to provide an effective anticoagulation of the extracorporeal blood circuit. Compared to systemic anticoagulation with heparin, RCA prolongs filter patency, allows to waive systemic anticoagulation, and reduces bleeding risk [1]. Thus, RCA is nowadays an accepted technique for all patients and not only for those at high risk of bleeding [2].
Austin Crit Care Case Rep - Volume 5 Issue 3 - 2021 Submit your Manuscript | www.austinpublishing group.com Khadzhynov et al. () All rights are reserved 
In addition, RCA might offer benefits in patients at risk to develop a Heparin-Induced Thrombocytopenia (HIT) because a systemic anticoagulation to prevent clotting of the CRRT circuit is not necessary. Thus, the risk of HIT is reduced in patients receiving RCA. By nature, RCA is of course not a substitute for systemic anticoagulation. Therefore, for general thrombosis prophylaxis a systemic anticoagulation is mandatory and in patients with HIT, alternative anticoagulants must be initiated immediately to avoid thromboembolic complications $[3,4]$.

In 2009, a RCA protocol for CRRT in CVVHD mode and variable treatment dose was published [5]. In a number of studies, this protocol was shown to be safe and effective with regard to the delivered dialysis dose, metabolic control as well as control of the acid-base status. In early studies, clotting-free filter survival was higher than $90 \%$ at 48 hours $[5,6]$. With growing experience, filter running time has increased even more. In a recent prospective multicentre study a clotting-free filter survival rate of $98 \%$ at 72 hours was observed [7]. Thus, filter-clotting has become a rare event with this RCA-CVVHD protocol $[8,9]$.

Nevertheless, during routine use of this RCA-CVVHD protocol in critically ill patients in the Intensive Care Unit (ICU) the observation emerged that a low but noticeable number of patients showed inexplicable recurrent early filter-clotting although the postfilter ionized calcium levels were in the target range. In some of these patients, repeated clotting occurred within a few hours after starting a new circuit. Recently, Lehner et al. reported a case of premature and recurrent filter clotting during RCA, which might have been caused by development of HIT in the patient [10].

Unexpected and repeated filter clotting during CRRT with RCA might foil the proven advantages of RCA. In this retrospective analysis, we, therefore, aimed to characterize patients and conditions related to recurrent early filter-clotting that was not due to obvious reasons as RCA-protocol violation or technical failure, and to investigate the efficacy of interventions to reduce the number of clotting events in such cases.

\section{Materials and Methods}

\section{Study design and study population}

The retrospective observational single-center study was performed at the University Hospital Charité, Berlin, Germany in six intensive care units. All adult patients treated with RCA-CVVHD were included in the study.

RCA-CRRT was performed as continuous veno-venous hemodialysis (RCA-CVVHD) using the Multifiltrate device with Ultraflux AV1000S dialyzer (Fresenius Medical Care, Bad Homburg, Germany) in all patients according to the previously published protocol [5,11,12]. Routine filter change was after 72 hours, as recommended by the manufacturer, with an allowed tolerance of \pm 12 hours for practical reasons.

\section{Detection of patients with recurrent early filter clotting}

Databases were screened for RCA-CVVHD treatments with at least two subsequent filter-clottings occurring within the first 48 hours of a filter or one clotting event within the first 8 hours. Cases with filter-clotting due to RCA-protocol violation or technical failure were excluded.

\section{Data collection}

Patients were identified and clinical data (patient demographics, laboratory parameters, CRRT parameters and concomitant medication) were collected from three different sources: a computerized billing database (SAP HANA, SAP Deutschland, Walldorf, Germany), the patient data management system used in the ICUs (Computer Organized Patient Report Assistant (COPRA), COPRA System GmbH, Sasbachwalden, Germany) and records of the daily prescriptions of renal replacement procedures.

Testing for HIT: Screening for anti-PF4/heparin antibodies was performed by lateral-flow-immunoassay (Milenia QuickLine HIT) and if positive confirmed by testing heparin-induced platelet aggregation (HIPA; in house).

For patients with recurrent filter-clotting the probability of HIT was calculated for the time-point of the occurrence of the first filterclotting retrospectively. Probability was estimated by $4 \mathrm{~T}$-scoring system [13].

\section{Statistical analysis}

Patient cohort characteristics and parameters were calculated as mean (and standard deviation [SD]) or, in case of non-parametric distribution of metric variables, as median (and interquartile range [IQR]). Differences were tested using t-tests for independent samples in case of normally distributed variables, using Mann-WhitneyTests for skewed variables. Significance level was $p=0.05$. Statistical analysis was performed with IBM SPSS Statistics Version 22 (IBM Corporation, USA).

\section{Results}

\section{Patients with recurrent early filter-clotting}

From September 2008 to January 2012 we treated 1183 patients with RCA-CCVHD. In this cohort, we identified 12 patients with recurrent early filter-clotting with a total of 147 filters used. The demographic data and clinical characteristics of these patients at time of ICU admission are shown in Table 1.

At the time of ICU admission, there were no known prothrombotic disorders in these patients, especially no history of HIT. Reasons for

Table 1: Characteristics of 12 patients with early recurrent filter clotting at time of ICU admission.

\begin{tabular}{|l|c|}
\hline Age (years) & $69(10)$ \\
\hline Male gender & $11(92 \%)$ \\
\hline Body weight $(\mathrm{kg})$ & $94(20)$ \\
\hline ESRD & $1(8 \%)$ \\
\hline SAPS II & $59(15)$ \\
\hline SOFA & $11(5)$ \\
\hline APACHE II at admission & $30(7)$ \\
\hline Sepsis & $12(100 \%)$ \\
\hline On vasopressor support & $12(100 \%)$ \\
\hline On mechanical ventilation & $11(92 \%)$ \\
\hline On extracorporeal membrane oxygenation & $1(8 \%)$ \\
\hline
\end{tabular}

Mean (SD) or rate (\%) is given. ESRD: Dialysis dependent end stage renal disease prior to ICU admission. 
Table 2: Laboratory values of 12 patients with recurrent early filter clotting at time of start of CRRT.

\begin{tabular}{|l|c|}
\hline Creatinine (mmol/L) & $211(70.4)$ \\
\hline aPTT (s) & $43(8)$ \\
\hline INR & $1.3(0.1)$ \\
\hline AT III (\%) & $68(16)$ \\
\hline Hemoglobin (g/dL) & $10.6(9.6-11.0)$ \\
\hline Hematocrit $(\%)$ & $31(30-34)$ \\
\hline Leucocyte $(/ \mathrm{nL})$ & $13.0(8.3-21.0)$ \\
\hline Lactate $(\mathrm{mg} / \mathrm{dL})$ & $23(13-37)$ \\
\hline Bilirubin $(\mathrm{mg} / \mathrm{dL})$ & $0.8(0.6-1.7)$ \\
\hline ALT $(\mathrm{U} / \mathrm{L})$ & $29(17-58)$ \\
\hline LDH $(\mathrm{U} / \mathrm{L})$ & $543(263-735)$ \\
\hline Urea $(\mathrm{mg} / \mathrm{dL})$ & $86(51-114)$ \\
\hline iCa $(\mathrm{mmol} / \mathrm{L})$ & $1.16(0.10)$ \\
\hline iCa postfilter $(\mathrm{mmol} / \mathrm{L})$ & $0.33(0.05)$ \\
\hline
\end{tabular}

aPTT: Activated Partial Thromboplastin Time; INR: International Normalized Ratio; AT III: Antithrombin III; ALT: Alanine Aminotransferase.

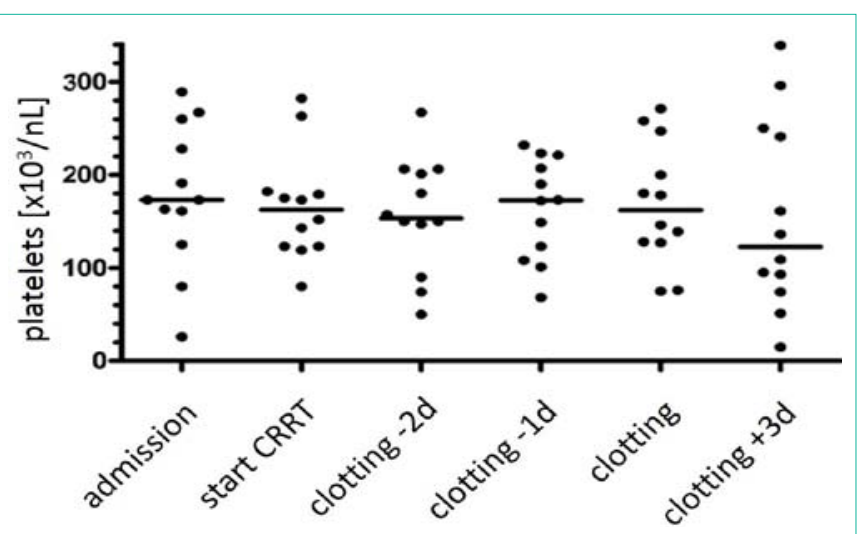

Figure 1: Platelet count of each patient with recurrent early filter-clotting at different time-points (at admission, start of RCA-CVVHD, 2 days and 1 day before clotting event, at day of clotting event, and 3 days after clotting event). There was no significant difference between individual counts at different time-points ( $p>0.310$ for all comparisons).

ICU admission were post- cardiac surgery in 10 cases, respiratory failure in exacerbated COPD in one case, and acute myocardial infarction in one case. One patient was dependent on hemodialysis due to end stage renal disease prior to ICU admission and was switched to CRRT in the ICU because of hemodynamic instability. All other patients had acute kidney injury (AKI) with need for CRRT. All patients had diagnosis of sepsis or septic shock.

Four patients (33\%) had received antifibrinolytic therapy (tranexamic acid) 10 to 3 days before the first clotting event. The time point of administration of tranexamic acid was not related to clotting events.

\section{RCA-CVVHD}

RCA-CVVHD was started in median (range) at 19 (10-29) hours after ICU admission. The patient's laboratory values at start of CRRT are shown in Table 2. All AKI patients were treated de novo with RCA-CVVHD. The patient with preexisting dialysis dependent ESRD was switched to RCA-CVVHD $30 \mathrm{~h}$ after admission at ICU without receiving intermittent dialysis. At initiation of RCACVVHD the mean $( \pm S D)$ blood flow was $107 \pm 10 \mathrm{~mL} / \mathrm{min}$, the mean $( \pm$ SD) dialysate flow was $2.1 \pm 0.2 \mathrm{~L} / \mathrm{h}$ corresponding to a mean $( \pm \mathrm{SD})$ prescribed dialysis dose of $23 \pm 3 \mathrm{~mL} / \mathrm{kg} / \mathrm{h}$, and the mean $( \pm \mathrm{SD})$ net ultrafiltration rate was $46 \pm 46 \mathrm{~mL} / \mathrm{h}$. The mean $( \pm \mathrm{SD})$ total duration of RCA-CVVHD therapy was $566 \pm 323$ hours. At time of first early filter-clotting, mean $( \pm \mathrm{SD})$ post-filter ionized calcium concentrations $(0.31 \pm 0.03 \mathrm{mmol} / \mathrm{L})$ were in the target range below $0.35 \mathrm{mmol} / \mathrm{L}$ in all patients.

\section{Heparin and argatroban administration}

All patients were systemically anticoagulated with unfractionated heparin or low-molecular weight heparin for at least 24 hours before initiation of RCA-CVVHD. At initiation of RCA-CVVHD all were switched to unfractionated heparin in a median dose of 2.7 (2.1-3.4) $\mathrm{U} / \mathrm{h} / \mathrm{kg}$.

\section{HIT}

At initiation of RCA-CVVHD, none of the patients had a diagnosis of HIT. During follow-up, 5 (42\%) of the 12 patients had a positive testing (HIPA) for hep-PF4-antibodies. In these patients, heparin was stopped and therapy with argatroban was started either before the results of the HIT testing were available due to suspected HIT, or immediately after testing positive for HIT. In patients with negative HIT testing argatroban therapy was started - for a variety of reasons - at different time points ranging from the day of ordering the HIT test to day nine but finally, all patients received argatroban.

At the time of first clotting, the $4 \mathrm{~T}$ score indicated a low probability of HIT in 9 patients (score 1-3) and a moderate probability in 3 patients (score 4-5). None of the patients had a score higher than 5 indicating high probability of HIT. Interestingly, in 3 out of 9 patients with low probability for HIT and early filter-clotting a follow-up test was positive, as well as in 2 out of 3 patients with moderate probability. The mean thrombocyte count was $185 \pm 71$ at the day of ICU admission, $172 \pm 52$ at RCA-CRRT initiation and $168 \pm 66$ at the first clotting event (Figure 1). At the first clotting event, none of the patients had clinical signs of thrombosis or thromboembolism.

From the 5 patients with positive testing for HIT, 3 patients received at least 19 days of effective argatroban treatment, one patient died after 8 days on treatment, and one case was lost to follow up after 3 days on treatment because of transfer to another hospital.

\section{Filter-clotting during heparin anticoagulation and after switch to argatroban}

The first unexplained early filter-clotting was observed after a median (range) of $102(0-136)$ hours after first initiation of RCACVVHD therapy.

The median interval from first application of heparin and/or low molecular weight heparin administration until first clotting event was 7.5 (6-13.5) days. Heparin was stopped and systemic anticoagulation was switched to argatroban after a mean of $82 \pm 75$ hours after the first clotting event.

After the first observed clotting event, 70 RCA-CVVHD sessions with systemic heparin administration and without argatroban therapy were compared to 58 sessions with continuous administration of intravenous argatroban. For estimation of filter lifetime, sessions with 


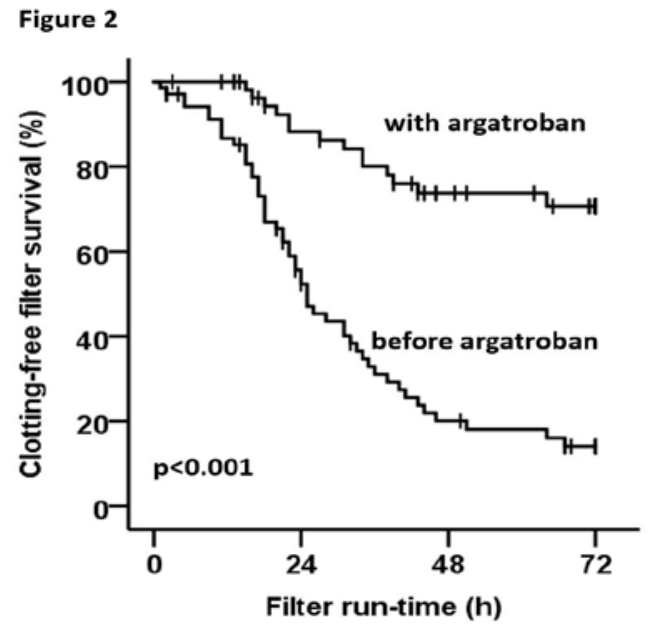

Figure 2: Kaplan-Meyer curves of the clotting-free filter survival before and after switch to argatroban therapy of all treatments (147) in 12 patients with recurrent early filter-clotting. Filter survival was censored for non-clotting events. The number at risk were 70 at $0 \mathrm{~h}, 33$ at $24 \mathrm{~h}, 11$ at $48 \mathrm{~h}$, and 4 at $72 \mathrm{~h}$ for sessions before switch to argatroban and 58 at $0 \mathrm{~h}, 44$ at $24 \mathrm{~h}, 28$ at $48 \mathrm{~h}$, and 20 at $72 \mathrm{~h}$ for sessions after switch to argatroban.

non-clotting events causing filter change were censored in the analysis of filter survival. Non-clotting events were defined as: transport to procedure, technical failure of vascular access or dialysis device, patient's death, switch to intermittent therapy, and renal recovery. The Kaplan-Meyer analysis of the clotting-free filter lifetime with or without argatroban of all patients is shown in Figure 2. After switch to systemic anticoagulation with argatroban the proportion of filters reaching a clotting free filter life-time of 72 hours increased from $14 \%$ to $71 \%$ (log-rank $\mathrm{p}<0.001$, Figure 2 ). The clotting-events during RCACVVHD treatment decreased from 0.61 to 0.10 clottings per $24 \mathrm{~h}$ of run-time. Early filter-clotting was prevented by switch to argatroban in filters of HIT-positive patients $(\mathrm{p}<0.001$, Figure $3 \mathrm{~A})$ and also in filters of HIT-negative patients ( $p=0.033$, Figure $3 \mathrm{~B}$ ). The effect of argatroban on clotting-free filter survival was significantly higher in patients tested positive for HIT ( $<<0.001$, Figure 3 C).

\section{Patient's outcome}

Three patients (two patients with positive HIT testing and one patient with negative HIT testing) developed peripheral venous thrombosis. Of those, one patient with positive HIT testing and thrombosis showed pulmonary embolism, which was diagnosed 2 days before initiation of argatroban and positive testing for HIT.

\section{Discussion}

In the present study, we investigated the underlying causes and conditions related to early recurrent filter-clotting during continuous veno-venous hemodialysis with regional citrate anticoagulation in patients with strict adherence to the protocol. The most important finding is that undetected HIT is present in a significant number of these patients, and that treatment with a direct thrombin inhibitor may prolong filter running time and reduce clotting events both in patients with and without proven HIT. Thus, besides HIT other undetected causes of thrombin generation may be present in these patients.

In critically ill patients on the ICU, the diagnosis of HIT is still a challenge because multiple causes alone or in combination make thrombocytopenia very common. Some frequently observed conditions are pseudothrombocytopenia, hemodilution, increased consumption, decreased production, increased sequestration, and immune-mediated destruction of platelets, but also bleeding events and surgical procedures [14]. In patients on renal replacement therapy, filter-clotting with consecutive blood loss may aggravate or cause thrombocytopenia per se. In summary, up to $50 \%$ of patients present with thrombocytopenia at some time point of their ICU stay, and $5 \%$ to $20 \%$ develop severe thrombocytopenia, defined as platelet counts $<50 \times 10^{9} / \mathrm{L}[15]$.

The most relevant result of the present analysis is that early filter clotting occurred in 5 patients in whom HIT was detected later on during the treatment course. Initially, i.e. at the time of the first unexpected clotting event, the $4 \mathrm{~T}$ scoring system classified them as patients with a low probability of HIT, and clinical signs of thrombosis/thromboembolism were not evident. Of note, it took 3 days from the first filter clotting until a significant drop in platelet count occurred which might stimulate clinicians to order a HIT testing. Therefore, to interpret early filter clotting in RCA-CVVHD as an early warning sign for a high probability for HIT might enhance the chance of earlier diagnosis and timely therapy of HIT in such patients.

In the present study, two subgroups of patients with early filterclotting were identified: one subgroup with positive laboratory test
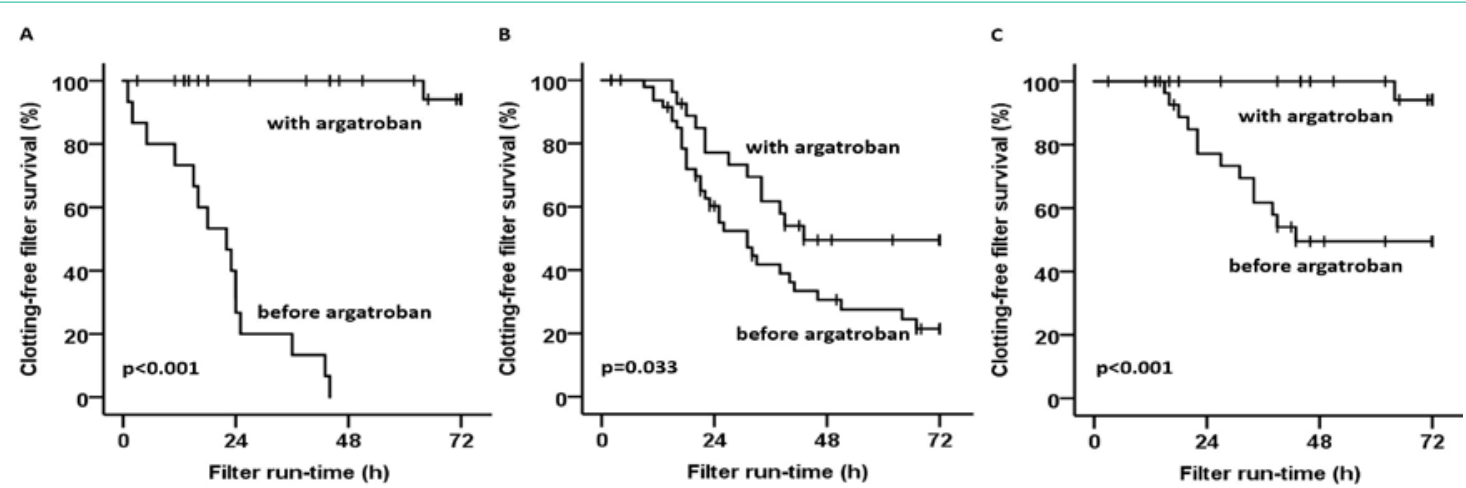

Figure 3: Kaplan-Meyer curves of clotting-free filter survival, censored for non-clotting events. A) Patients tested positive for HIT-II before and after switch to argatroban. B) Patients tested negative for HIT-II before and after switch to argatroban. C) After switch to argatroban, patients tested negative or positive for HIT-II. 
for HIT and marked improvement of filter run-time after start of argatroban, and another subgroup with negative test for HIT and a significant, but less pronounced prolongation of filter run-time after treatment with argatroban $(\mathrm{p}=0.033)$. In the latter group, obviously other factors than HIT must have caused systemic thrombin generation. Of note and as a common feature, all patients were in sepsis or septic shock. In early sepsis, activation of the coagulation system is triggered by proinflammatory cytokines that enhance the expression of tissue factor on activated mononuclear and endothelial cells and simultaneously downregulate natural anticoagulants, thus initiating thrombin generation, subsequent activation of platelets, and inhibition of fibrinolysis [16]. This might explain in part our observations in those without proven HIT. Unfortunately, due to the retrospective character of the study, additional testing to evaluate the exact underlying mechanism for early filter-clotting was not possible.

The mode of anticoagulation by citrate as suggested below is consistent with the observation that in conditions with systemic thrombin generation - as it is also part of HIT - citrate anticoagulation alone is not sufficient to achieve adequate anticoagulation in the extracorporeal circuit so that early clotting may occur. It is well-known that calcium ions are essential at various steps of the clotting cascade [17]. Besides other functions, calcium is involved in the interaction with the Gamma-Carboxylated Glutamic Acid (GLA) domain of the Vitamin K-dependent clotting factors, which include Prothrombin, Factor VII, Factor IX, Factor X [18]. Physiologically, calcium ions are integrated into the GLA domain of the vitamin K-dependent clotting-factors, leading to structural changes of the clotting-factor [19] which facilitate binding to an activated phospholipid-membrane [20] and initiate coagulation.

The calcium binding sites of the GLA domain have been reported as being of intermediate strength, with a binding constant of about $0.45 \mathrm{mmol} / 1[21]$. Of note, this binding constant is slightly above the target range for the post-filter ionized calcium target during RCA, i.e. $0.25-0.35 \mathrm{mmol} / \mathrm{l}[5,9]$. Thus, it is the transient dissociation of calcium from the GLA domain of key clotting factors, which prevents the propagation of clotting processes at various steps, if GLA domain containing clotting factors are involved.

Different to other activated serine proteases (F VIIa, F IXa, F Xa, activated protein $\mathrm{C}$ ), the GLA domain contained in prothrombin is removed during the activation process from prothrombin to thrombin. Thus, the active thrombin does not further contain a GLA domain, which means that the activity of thrombin is independent of GLA domains and calcium. In consequence, the above suggested mode of anticoagulation by citrate does not influence the thrombin activity, once it is created in the systemic blood. Additionally, key reactions catalyzed by thrombin, i.e. conversion of fibrinogen to fibrin and activation of factor XIII are not calcium depending and the crosslinking of fibrin by activated factor XIII takes place at very low calcium concentrations. In consequence, RCA is most likely insufficient to prevent filter-clotting during clinical conditions leading to systemic thrombin generation, such as HIT. Therefore, with regard to prevention of filter-clotting, thrombin has to be inhibited by administration of an adequate anticoagulant, e.g. argatroban.

There are several limitations of the present study. At first, due to the retrospective design of the study, the true incidence of patients with recurrent early filter clotting most likely linked to systemic thrombin generation might be underestimated. At second, although we carefully studied all documentations, we might have missed some technical problems which might have contributed to early clotting. Finally, since it is not possible to perform specific testing of coagulation parameters in a retrospective study setting, the theory of the underlying mechanism for early filter clotting has a sound pathophysiological background but should be investigated prospectively in a more detailed fashion.

\section{Conclusion}

Early and otherwise unexplained filter-clotting in RCA-CVVHD patients who receive additional systemic heparin can be a first and early warning sign of HIT. In those patients, immediate testing for HIT should be initiated and a switch from heparin to a direct thrombin inhibitor like argatroban can be recommended. In patients with HIT as well as in those without HIT but activated thrombin due to other reasons the application of a direct thrombin inhibitor like argatroban significantly improves filter run-times of the RCACVVHD therapy.

\section{Declaration}

Acknowledgement: Besides our obligation to thank all patients included in the study, we are devoting this manuscript with deep sadness to our beloved friend and colleague, Torsten Slowinski. His unexpected passing during the preparation of the manuscript left an empty space in our hearts.

Statement of Ethics: All study procedures were approved by the institutional ethics committee (Ethikkommission der ChariteUniversitätsmedizin Berlin; EA1/035/12) ensuring adherence to the Declaration of Helsinki and accordance to the guidelines of securing good scientific practice. Upon approval by the local ethical review committee, the need for patients' informed consent was waived because of the observational nature of the study, the absence of any intervention, and the anonymization of all data sets used for analysis.

Conflict of Interest Statement: Dr. Khadzhynov, Dr. Slowinski and Dr. Kindgen-Milles have received funds for speaking at symposia organized on behalf of Fresenius Medical Care, Bad Homburg, Germany. The other authors have nothing to declare.

Author Contributions: Dr. Slowinski and Dr. Staeck designed the study. Dr. Khadzhynov, Dr. Schreiber and Dr. Lieker contributed to study design, data collection, data analyses and interpretation. Dr. Schreiber performed data collection. Drs. Eckardt, Budde, Kindgen-Milles, Halleck and Lehner contributed to study design and interpretation. Dr. Staeck performed statistical analyses. Drs. Slowinski, Staeck and Khadzhynov wrote the first draft of the manuscript and revised subsequent versions. All authors read and approved the final manuscript.

\section{References}

1. Bai M, Zhou M, He L, Ma F, Li Y, Yu Y, et al. Citrate versus heparin anticoagulation for continuous renal replacement therapy: an updated metaanalysis of RCTs. Intensive Care Med. 2015; 41: 2098-2110.

2. Kidney Disease: Improving Global Outcomes (KDIGO) Acute Kidney Injury Work Group. KDIGO Clinical Practice Guideline for Acute Kidney Injury. Kidney Int. 2012: 1-138.

3. Oudemans-van Straaten HM, Kellum JA, Bellomo R. Clinical review: 
anticoagulation for continuous renal replacement therapy--heparin or citrate? Crit Care. 2011; 15: 202.

4. Brandenburger T, Dimski T, Slowinski T, Kindgen-Milles D. Rena replacement therapy and anticoagulation. Best Pract Res Clin Anaesthesiol. 2017; 31: 387-401.

5. Morgera S, Schneider M, Slowinski T, Vargas-Hein O, Zuckermann-Becker $\mathrm{H}$, Peters $\mathrm{H}$, et al. A safe citrate anticoagulation protocol with variable treatment efficacy and excellent control of the acid-base status. Crit Care Med. 2009; 37: 2018-2024.

6. Kalb R, Kram R, Morgera S, Slowinski T, Kindgen-Milles D. Regional citrate anticoagulation for high volume continuous venovenous hemodialysis in surgical patients with high bleeding risk. Ther Apher Dial. 2013; 17: 202-212.

7. Slowinski T, Morgera S, Joannidis M, Henneberg T, Stocker R, Helset E et al. Safety and efficacy of regional citrate anticoagulation in continuous venovenous hemodialysis in the presence of liver failure: the Liver Citrate Anticoagulation Threshold (L-CAT) observational study. Crit Care. 2015; 19 : 349 .

8. Khadzhynov D, Dahlinger A, Schelter C, Peters H, Kindgen-Milles D, Budde K et al. Hyperlactatemia, Lactate Kinetics and Prediction of Citrate Accumulation in Critically III Patients Undergoing Continuous Renal Replacement Therapy With Regional Citrate Anticoagulation. Crit Care Med. 2017; 45: e941-e946.

9. Khadzhynov D, Deissler J, Bobonov O, Schelter C, Peters H, Kindgen-Milles $\mathrm{D}$, et al. Intensive monitoring of post filter ionized calcium concentrations during CVVHD with regional citrate anticoagulation: A retrospective study. $\mathrm{J}$ Crit Care. 2020; 58: 1-5.

10. Lehner GF, Schopf M, Harler U, Pechlaner C, Joannidis M. Repeated premature hemofilter clotting during regional citrate anticoagulation as indicator of heparin induced thrombocytopenia. Blood Purif. 2014; 38: 127 130 .

11. Kindgen-Milles D, Amman J, Kleinekofort W, Morgera S. Treatment of metabolic alkalosis during continuous renal replacement therapy with regional citrate anticoagulation. Int J Artif Organs. 2008; 31: 363-366.

12. Khadzhynov D, Schelter C, Lieker I, Mika A, Staeck O, Neumayer HH, et al
Incidence and outcome of metabolic disarrangements consistent with citrate accumulation in critically ill patients undergoing continuous venovenous hemodialysis with regional citrate anticoagulation. J Crit Care. 2014; 29: 265271.

13. Warkentin TE, Greinacher A, Gruel Y, Aster RH, Chong BH, scientific, et al. Laboratory testing for heparin-induced thrombocytopenia: a conceptual framework and implications for diagnosis. J Thromb Haemost. 2011; 9: 24982500.

14. Greinacher A, Selleng S. How I evaluate and treat thrombocytopenia in the intensive care unit patient. Blood. 2016; 128: 3032-3042.

15. Hui P, Cook DJ, Lim W, Fraser GA, Arnold DM. The frequency and clinical significance of thrombocytopenia complicating critical illness: a systematic review. Chest. 2011; 139: 271-278.

16. Fourrier F. Severe sepsis, coagulation, and fibrinolysis: dead end or one way? Crit Care Med. 2012; 40: 2704-2708.

17. Calatzis A, Toepfer M, Schramm W, Spannagl M, Schiffl H. Citrate anticoagulation for extracorporeal circuits: effects on whole blood coagulation activation and clot formation. Nephron. 2001; 89: 233-236.

18. Skogen WF, Bushong DS, Johnson AE, Cox AC. The role of the Gla domain in the activation of bovine coagulation factor $X$ by the snake venom protein XCP. Biochem Biophys Res Commun. 1983; 111: 14-20.

19. Whinna HC, Lesesky EB, Monroe DM, High KA, Larson PJ, Church FC. Role of the gamma-carboxyglutamic acid domain of activated factor $\mathrm{X}$ in the presence of calcium during inhibition by antithrombin-heparin. J Thromb Haemost. 2004; 2: 1127-1134.

20. Falls LA, Furie BC, Jacobs M, Furie B, Rigby AC. The omega-loop region of the human prothrombin gamma-carboxyglutamic acid domain penetrates anionic phospholipid membranes. J Biol Chem. 2001; 276: 23895-23902.

21. Sabharwal AK, Padmanabhan K, Tulinsky A, Mathur A, Gorka J, Bajaj $\mathrm{SP}$. Interaction of calcium with native and decarboxylated human factor $X$ Effect of proteolysis in the autolysis loop on catalytic efficiency and factor $\mathrm{Va}$ binding. J Biol Chem. 1997; 272: 22037-22045. 